Article

\title{
A Low-Noise X-ray Astronomical Silicon-On-Insulator Pixel Detector Using a Pinned Depleted Diode Structure
}

\author{
Hiroki Kamehama ${ }^{1}$, Shoji Kawahito ${ }^{2, *}$, Sumeet Shrestha ${ }^{2}$, Syunta Nakanishi ${ }^{2}$, \\ Keita Yasutomi ${ }^{2}$, Ayaki Takeda ${ }^{3}$, Takeshi Go Tsuru ${ }^{4}$ and Yasuo Arai ${ }^{5}$ \\ 1 Information and Communication Systems Engineering, National Institute of Technology, Okinawa College, \\ Okinawa 905-2171, Japan; hkame@okinawa-ct.ac.jp \\ 2 Research Institute of Electronics, Shizuoka University, Shizuoka 432-8011, Japan; \\ sumeet@idl.rie.shizuoka.ac.jp (S.S.); snakani@idl.rie.shizuoka.ac.jp (S.N.); kyasu@idl.rie.shizuoka.ac.jp (K.Y.) \\ 3 Department of Applied Physics and Electronic Engineering, University of Miyazaki, Miyazaki 889-2192, \\ Japan; takeda@astro.miyazaki-u.ac.jp \\ 4 Department of Physics, Kyoto University, Kyoto 606-8502, Japan; tsuru@cr.scphys.kyoto-u.ac.jp \\ $5 \quad$ High Energy Accelerator Research Organization, Tsukuba, Ibaraki 305-0801, Japan; yasuo.arai@kek.jp \\ * Correspondence: kawahito@idl.rie.shizuoka.ac.jp; Tel.: +81-53-478-1313
}

Received: 1 November 2017; Accepted: 20 December 2017; Published: 23 December 2017

\begin{abstract}
This paper presents a novel full-depletion Si X-ray detector based on silicon-on-insulator pixel (SOIPIX) technology using a pinned depleted diode structure, named the SOIPIX-PDD. The SOIPIX-PDD greatly reduces stray capacitance at the charge sensing node, the dark current of the detector, and capacitive coupling between the sensing node and SOI circuits. These features of the SOIPIX-PDD lead to low read noise, resulting high X-ray energy resolution and stable operation of the pixel. The back-gate surface pinning structure using neutralized p-well at the back-gate surface and depleted n-well underneath the p-well for all the pixel area other than the charge sensing node is also essential for preventing hole injection from the p-well by making the potential barrier to hole, reducing dark current from the $\mathrm{Si}-\mathrm{SiO}_{2}$ interface and creating lateral drift field to gather signal electrons in the pixel area into the small charge sensing node. A prototype chip using $0.2 \mu \mathrm{m}$ SOI technology shows very low readout noise of $11.0 \mathrm{e}^{-}$rms, low dark current density of $56 \mathrm{pA} / \mathrm{cm}^{2}$ at $-35{ }^{\circ} \mathrm{C}$ and the energy resolution of $200 \mathrm{eV}$ (FWHM) at $5.9 \mathrm{keV}$ and $280 \mathrm{eV}$ (FWHM) at $13.95 \mathrm{keV}$.
\end{abstract}

Keywords: SOI X-ray detector; high energy resolution; low noise; back-side surface potential pinning structure

\section{Introduction}

$\mathrm{X}$-ray astronomical satellites require low-noise high-time-resolution high-spatial-resolution detectors. X-ray charge-coupled devices (CCDs) are currently used as the standard imaging devices because they offer Fano-limited spectroscopic performance $(\sim 120 \mathrm{eV}$ in FWHM at $6 \mathrm{keV})$ with a low readout noise of about $3 \mathrm{e}^{-}$rms [1,2]. However, $X$-ray CCDs suffers from the poor time resolution (a few seconds) and the low dynamic range (0.3-10 keV) [3]. To meet the requirements of both low noise and high time-resolution, complementary metal oxide semiconductor (CMOS)-based event-driven type of detectors are being developed. One of techniques to implement CMOS event-driven detectors is to use a hybrid detector structure which uses stacking of a silicon detector and CMOS readout electronics through Indium bump interconnections [4]. However, hybrid CMOS detectors have a limitation on large pixel number, small pixel size, and high production yield. Another technique for CMOS event-driven detectors is to use monolithic CMOS detector technology. Monolithic CMOS detectors do not require any mechanical bump bonding between detector and readout circuits. Pixel 
size can be relatively small for higher spatial resolution. The silicon-on-insulator pixel (SOIPIX) detector technology being developed by the High Energy Accelerator Research Organization (KEK, Ibaraki, Japan) and Lapis Semiconductor, Inc. (Kanagawa, Japan) is a good platform for implementing a monolithic CMOS event-driven detector [3]. The SOIPIX is an active pixel sensor based on a semiconductor pixel detector realized with a CMOS fully depleted (FD-) SOI technology.

Figure 1 shows the cross-sectional view of the SOIPIX. The SOI wafer is composed of a thick, high-resistivity substrate for the sensing part and a thin Si layer for CMOS circuits sandwiched by a buried oxide (BOX) layer. The SOIPIX utilizes a buried p-well (BPW) as a sensing part to detect X-rays [5]. Using the $0.2 \mu \mathrm{m}$ CMOS fully depleted (FD) SOI technology, we have been developing an event-driven X-ray pixel (XRPIX) series [6-9]. Though their basic characteristics have been gradually improved, there still are issues on the improvements of noise, charge correction efficiency, and the resulting X-ray energy resolution. The latest version of XRPIX called XRPIX3b uses a charge-sensitive amplifier (CSA) circuit in each pixel to increase the conversion gain and reduce readout noise. The XRPIX3b has achieved the readout noise of $35 \mathrm{e}^{-}$rms and the energy resolution of $320 \mathrm{eV}(\mathrm{FWHM})$ at $6 \mathrm{keV}$ [9]. The detector structure used for the XRPIX3b, however, has a difficulty of reduction of the sensing-node capacitance, a problem of crosstalk between the sensing node and SOI CMOS circuits, and dark current generation at the $\mathrm{Si}_{-} \mathrm{SiO}_{2}$ interface under the BOX. To solve the crosstalk problem, an SOIPIX technology using the nested-well structure has been proposed [10]. Though this technique is effective for reducing the crosstalk problem, the nested-well structure still has an issue on the large capacitance at the sensing node. The SOIPIX using double SOI layers recently reported is an attractive device for realizing a low-noise detector that exploits the merits of the middle Si layer (middle SOI) for reducing the crosstalk by shielding the sensing node from the SOI circuits and reducing the sensing-node capacitance with the structure of the depleted substrate Si surface ( $\mathrm{Si}-\mathrm{SiO}_{2}$ interface under the BOX layer) [11]. However, it still has issues on dark current generation at the depleted substrate Si surface, and a possibility of signal charge loss by the traps at the Si surface, leading to the degraded charge collection efficiency.

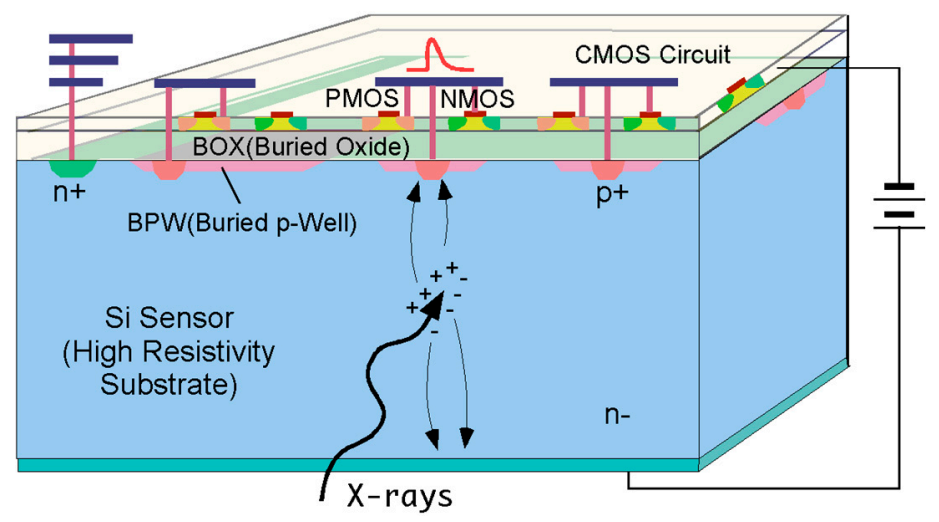

Figure 1. The cross-sectional view of the SOIPIX.

In order to realize an X-ray pixelated detector with high energy resolution based on the SOIPIX technology, this paper proposes a novel SOIPIX using a pinned depleted diode structure. This pixel technology called the SOIPIX-PDD allows us to solve the issues of conventional SOIPIXs associated with the readout noise, dark current, crosstalk, and charge collection efficiency, while having a feature of fully depleted thick sensing region of the handle substrate which is commonly required for high energy imaging [12-14]. Thanks to the pinned depleted diode structure having features of the pinned $\mathrm{Si}$ surface layer which also acts as an electro-static shielding layer and depleted buried channel for carrier collection to the small-capacitance charge sensing node, dark current at substrate Si surface, readout noise and crosstalk are greatly reduced. This structure is also effective for high charge collection efficiency and high-speed response because the signal carriers collected run in the buried channel 
with the help of lateral electro-static field but without touching to the Si surface. The rest of this paper describes the pixel device structure, pixel circuits, implementation and evaluation results of the SOIPIX-PDD, and finally conclusions.

\section{SOI Pixel Detector Using a Pinned Depleted Diode Structure}

\subsection{Sensor Structure Implemented on High-Resistivity Substrate}

In the conventional SOIPIX as shown in Figure 1, the charge sensing node is made with a BPW at the back-gate surface of the high-resistivity $\mathrm{Si}$ substrate and the other part of back-gate surface is depleted. This depleted back-gate surface leads to a large dark current and charge loss due to the interface states. To increase the charge collection efficiency, the size of detector BPW must be increased and the resulting capacitance of the detector is increased, leading to a large capacitance of the sensing node. The capacitive coupling between the SOI circuits and the buried BPW may cause an additional noise and offset. To reduce the capacitive coupling between the sensing node (BPW) and SOI circuits, a nested-well structure based on the SOIPIX has been proposed [10,15]. However, the nested-well structure uses a large-size sensing plate made with neutralized BPW created underneath a buried n-well (BNW) and the capacitance of the sensing node becomes pretty large.

The SOIPIX using the pinned depleted diode structure, the SOIPIX-PDD, is developed to improve the detector performance compared with the conventional SOI pixel detector while maintaining the fundamental merit of the SOIPIX [16]. Figure 2 shows the cross-sectional view of the SOIPIX-PDD. For X-ray imaging, high negative voltage is applied at the backside of the detector for attaining a fully depleted thick substrate. A BPW is formed on the backside of the BOX for pinning the back-gate voltage of the SOI transistors to a fixed bias of $\mathrm{V}_{\mathrm{BB} 2}$. The BPW acts as a shielding layer between the charge sensing node and the SOI circuits, preventing the extra noise and offset generation by the coupling.

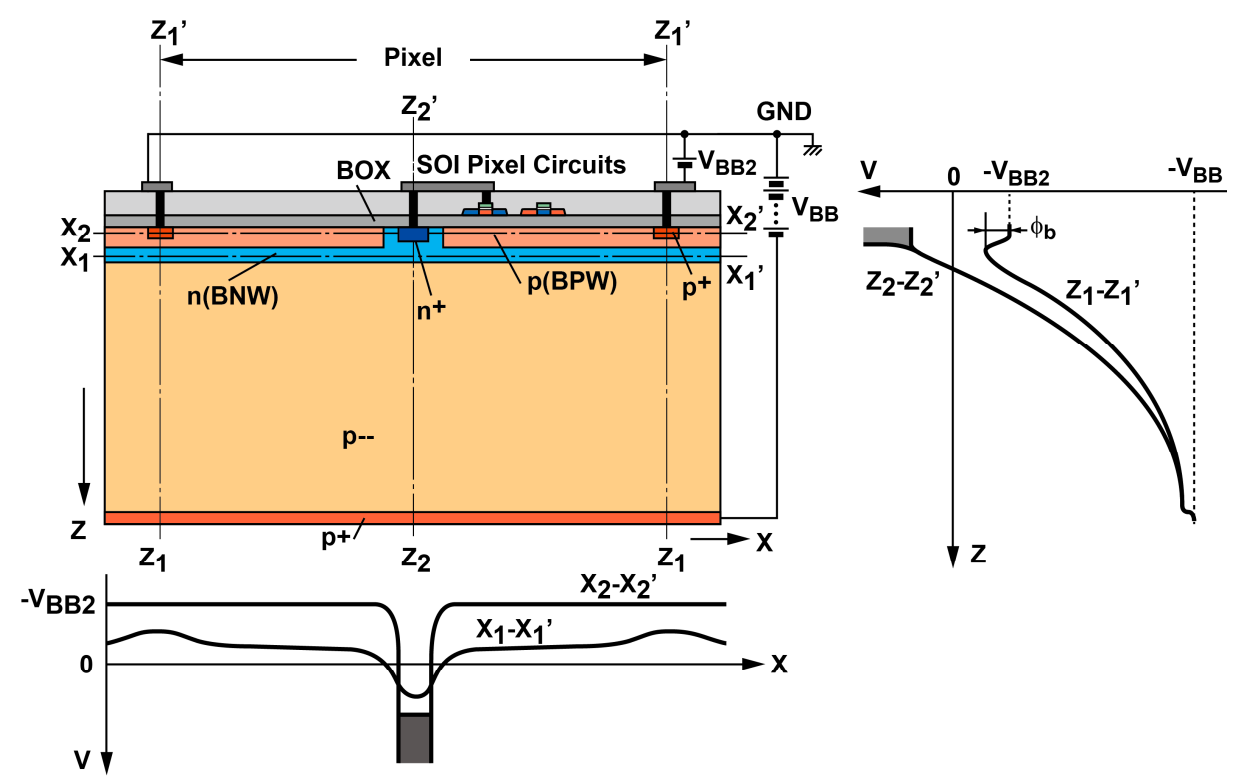

Figure 2. Cross-sectional view of the SOIPIX-PDD.

The sufficiently highly-doped BPW as a neutral region is effective for reducing the dark current generation at $\mathrm{Si}_{-} \mathrm{SiO}_{2}$ interface under the $\mathrm{BOX}$, because it works like a pinned photodiode in CCD or CMOS image sensors [17]. A BNW formed under the BPW is depleted and this layer acts as a buried channel to gather carriers generated in the pixel into the sensing node $(n+)$ and to improve charge collection efficiency, because lateral electric field is created in this channel as shown in the 
potential profile of $\mathrm{X}_{1}-\mathrm{X}_{1}{ }^{\prime}$ and a problem of the carrier trapping at $\mathrm{Si}-\mathrm{SiO}_{2}$ interface under the $\mathrm{BOX}$ in the conventional SOIPIX is solved by the buried channel structure. One important design issue is to minimize the leakage current from the BPW to the back-side $\mathrm{p}+$ layer by creating a sufficient potential barrier $\varphi_{\mathrm{b}}$ to holes as shown in Figure 2.

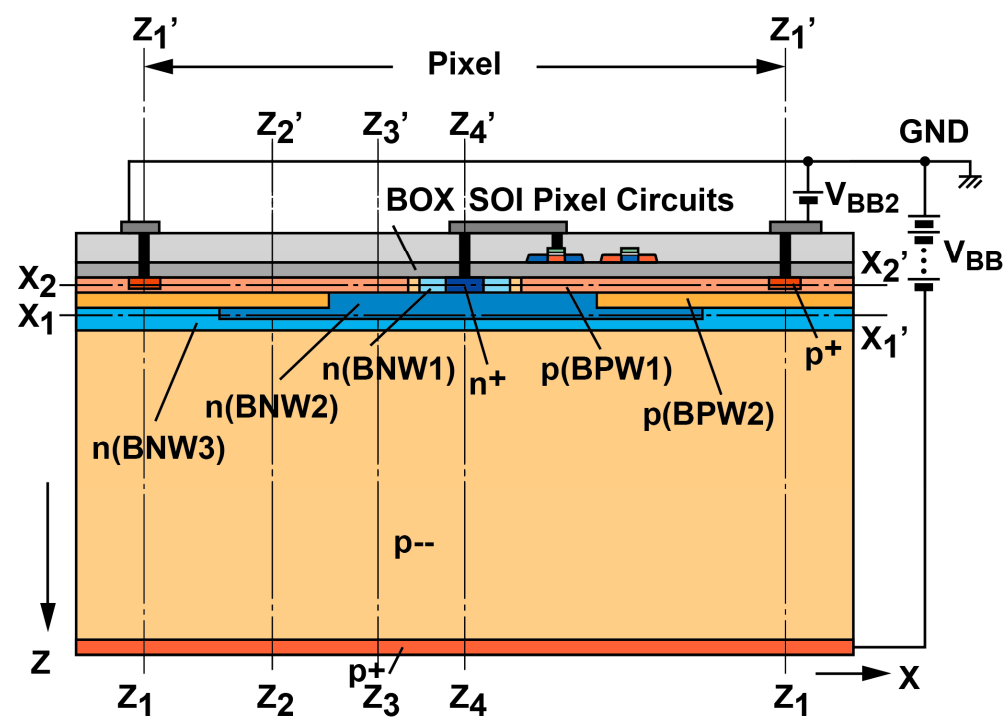

Figure 3. Cross-sectional view of the SOIPIX-PDD with multiple buried wells.

The SOIPIX-PDD shown in Figure 2 uses a single BNW for creating a channel with lateral electric field. If the pixel size is very large, the electric field created by this BNW only may not be sufficient for gathering charges within a time to meet the required X-ray photon incidental rate of $>1 \mathrm{MHz}$. In order to create a sufficiently large lateral electric field in whole detector volume, a multiple buried-well structure for the PDD as shown in Figure 3 is used. In this detector, the lateral electric field in the channel is created by two buried p-well (BPW1 and BPW2) and three buried n-well (BNW1, BNW2 and BNW3) are used. Since the BNW3, BNW2 and BNW1 under the BPW are depleted, the charge sensing capacitance of the detector is only due to the PN junction between the BPW and $n+$ and a part of the BNW near n+, a high charge-to-voltage conversion gain and the resulting low readout noise are realized.

\subsection{Simulation of Potential Profiles of the Designed SOIPIX-PDD}

Based on the pinned depleted diode with the multiple buried wells, a pixel detector for X-ray energy spectrum measurements is designed and its potential profiles are simulated by a device simulator SPECTRA.

Figure 4 shows the pixel layout pattern of the detector. The pixel size of the detector is $36 \mu \mathrm{m} \times 36 \mu \mathrm{m}$. A p+ layer is formed at the boundary of the pixel to bias the BPW1 for pinning the back-gate of the SOI circuits to $\mathrm{V}_{\mathrm{BB} 2}$. The pattern edges of the multiple buried p-/n-wells are located at $1.5 \mu \mathrm{m}$ for BNW1 (octagonal, positive tone), at $2.7 \mu \mathrm{m}$ for BPW1 (octagonal, negative tone), at $9 \mu \mathrm{m}$ for BPW2 (octagonal, negative tone), $13.5 \mu \mathrm{m}$ for BNW2 (octagonal, positive tone). The BNW3 covers all the pixel area $(36 \mu \mathrm{m} \times 36 \mu \mathrm{m})$. The thickness of the sensor layer (p-type substrate) is $200 \mu \mathrm{m}$. The voltages applied at the sensing node $(\mathrm{n}+)$, the back-gate of the SOI (p+, and BPW1), and the substrate backside $\mathrm{p}+\left(\mathrm{V}_{\text {back }}\right)$ are set to $3 \mathrm{~V},-4 \mathrm{~V}\left(=\mathrm{V}_{\mathrm{BB} 2}\right)$ and $-15 \mathrm{~V}\left(=\mathrm{V}_{\mathrm{BB}}\right)$, respectively. 


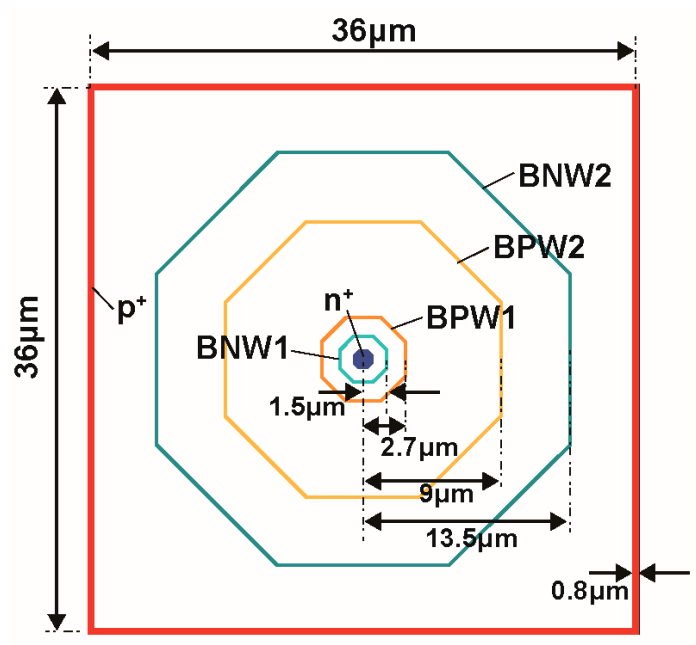

Figure 4. Sensor-layer patterns and dimensions of buried n-/p-wells.

Figure 5 shows the simulated potential profiles of the designed SOIPIX-PDD with multiple buried wells. Figure $5 a, b$ show the potential distribution of vertical cross-sections along $Z_{1}-Z_{1}{ }^{\prime}, Z_{2}-Z_{2}{ }^{\prime}, Z_{3}-Z_{3}{ }^{\prime}$ and $Z_{4}-Z_{4}{ }^{\prime}$ of Figure 3. The entire sensor layer is fully depleted from the surface to the backside. Figure $5 \mathrm{~b}$ is a zoomed potential distribution from the depth of the surface to $10 \mu \mathrm{m}$. In the cross-section of $Z_{4}-Z_{4}{ }^{\prime}$, a potential profile that carriers generated in the deep inside of silicon is directly transferred to the $n+$ sensing node. In the cross-sections of $Z_{1}-Z_{1}{ }^{\prime}, Z_{2}-Z_{2}{ }^{\prime}$ and $Z_{3}-Z_{3}{ }^{\prime}$, the back-gate surface is pinned to the applied voltage $(=-4 \mathrm{~V})$ to the BPW1, while creating a potential barrier $\varphi_{\mathrm{b}}$ of larger than $2 \mathrm{~V}$, which is sufficiently large to prevent hole injection from the BPW1. As shown in Figure 5b, the actual potential of the neutral region of the BPW1 $(=-4.4 \mathrm{~V})$ includes Fermi potential of $-0.4 \mathrm{~V}$. In the cross-sections of $Z_{2}-Z_{2}{ }^{\prime}, Z_{3}-Z_{3}{ }^{\prime}$ and $Z_{4}-Z_{4}{ }^{\prime}$, the carriers generated at deep inside of silicon is once coming to the near surface (buried channel) and then horizontally transferred to the $n+$ sensing node through the channel. Figure 6 shows the horizontal potential profiles at the cross-sections of $\mathrm{X}_{1}-\mathrm{X}_{1}{ }^{\prime}$ and $\mathrm{X}_{2}-\mathrm{X}_{2}{ }^{\prime}$. The potential profile of $\mathrm{X}_{2}-\mathrm{X}_{2}{ }^{\prime}$ shows the back-gate (BPW1) is pinned to $-4.4 \mathrm{~V}$. As shown in the potential of $\mathrm{X}_{1}-\mathrm{X}_{1}{ }^{\prime}$, lateral electric field is formed to collect photoelectrons in the pixel to the $\mathrm{n}+$ sensing node. Figure 7 is a 2-D potential plot at near the $S i$ substrate surface ( $Z=0$ to $10 \mu \mathrm{m}$ ). The simulated 2-D potential plot of the SOIPIX-PDD using multi-well structure shows that all the electrons generated from the surface to bottom of the pixel are gathered to the $n+$ sensing node through the depleted 3-D potential profile (X-Y-Z) of the detector, realizing high charge collection efficiency which is indicated by the shape of potential profile that collect carriers generated in the entire 3-D volume of the pixel into the $\mathrm{n}+$ sensing node.

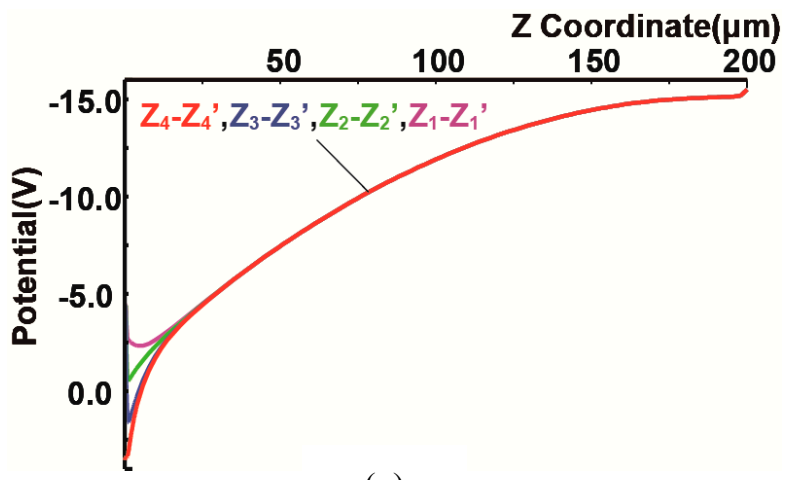

(a)

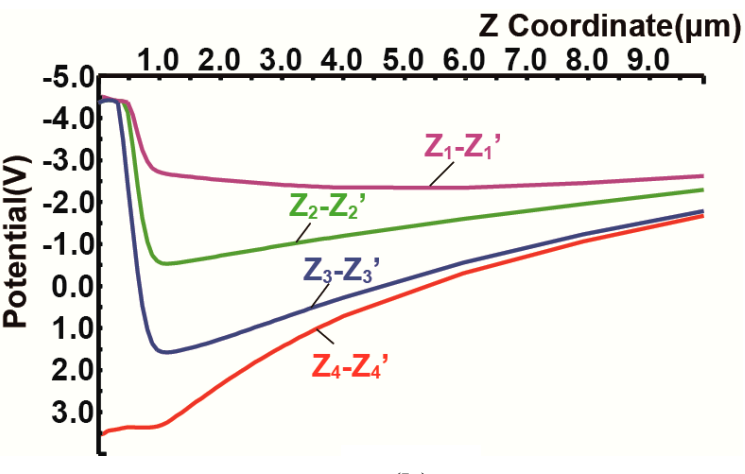

(b)

Figure 5. Simulated Vertical Potential Profiles, (a) $Z=0$ to $200 \mu \mathrm{m},(\mathbf{b}) Z=0$ to $10 \mu \mathrm{m}$. 


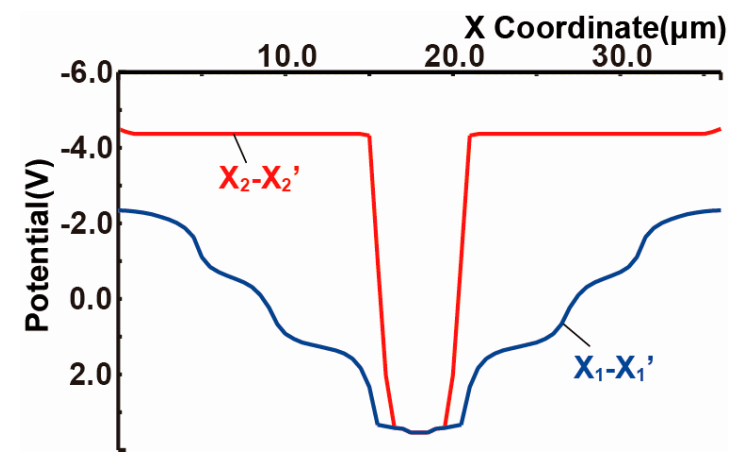

Figure 6. Simulated Horizontal Potential Profiles.

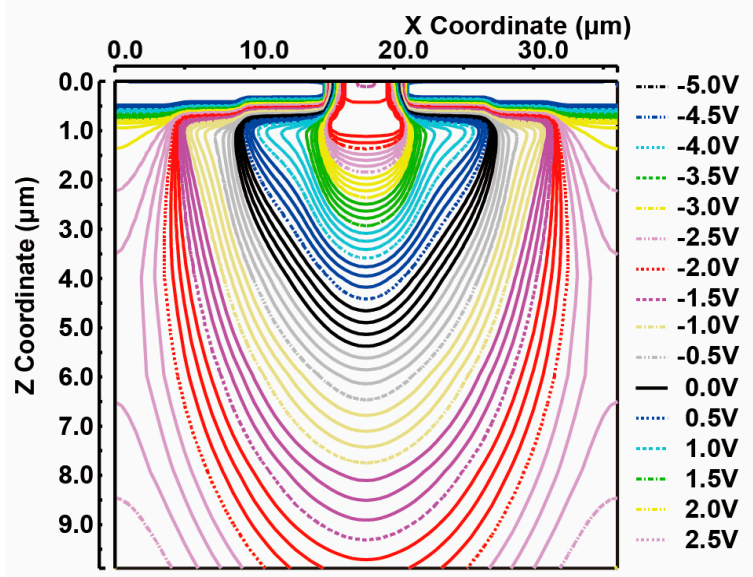

(a)

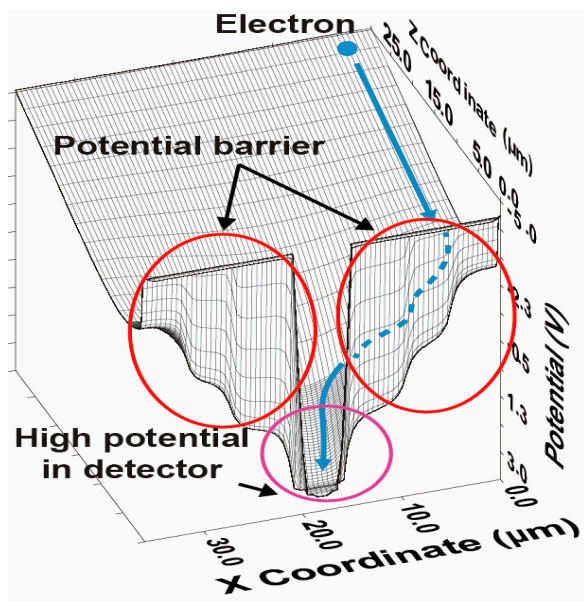

(b)

Figure 7. 2-D (X-Z) Equipotential plot (a) and Bird's Eye View of the 2-D (X-Z) Potential (b).

The capacitance of the $\mathrm{n}+$ sensing node denoted by $C_{D}$ can be estimated by the quasi-Fermi level change and the resulting change of accumulated electrons. The capacitance of $C_{D}$ is given by:

$$
C_{D}=\frac{Q_{\text {sig }}}{\Delta V_{\text {Nfermi }}}
$$

where, $Q_{\text {sig }}$ is quasi-accumulated electrons. From the simulation results of Figure $8, C_{D}$ is estimated to be $C_{D}=\left(q \Delta N_{\text {sig }}\right) / \Delta V_{N f e r m i}=\left(1.602 \times 10^{-19} \times 19,900\right) / 1.0 \cong 3.2 \mathrm{fF}$.

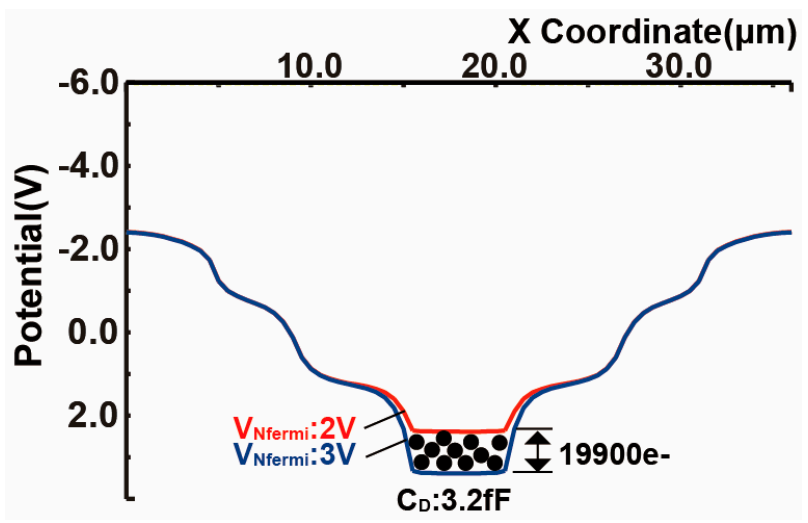

Figure 8. Estimation of $C_{D}$. 


\section{Charge-Sensitive Amplifier Design for Low-Noise Pixelated Detectors}

Figure 9 shows equivalent circuits of the charge-sensitive amplifier (CSA) in the pixel including the model of the SOI substrate detector. In the SOIPIX-PDD, the SOI substrate detector is modeled with two diodes, $D_{1}$ and $D_{2}$, the stray capacitance $C_{D}$ at the $n+$ charge sensing node of the substrate detector. The charge-to-voltage conversion gain of the CSA is given by

$$
G_{C}=q \frac{G_{A M P}}{C_{D}+C_{I}+C_{F B} \cdot G_{A M P}}
$$

where $C_{I}$ is the input capacitance of the internal amplifier, $C_{F B}$ is the feedback capacitance of the CSA, $G_{A M P}$ is the DC open-loop gain of the internal amplifier, and q is the elementary charge. If $G_{A M P}>>1$, it is approximated as:

$$
G_{C}=\frac{q}{C_{F B}}
$$

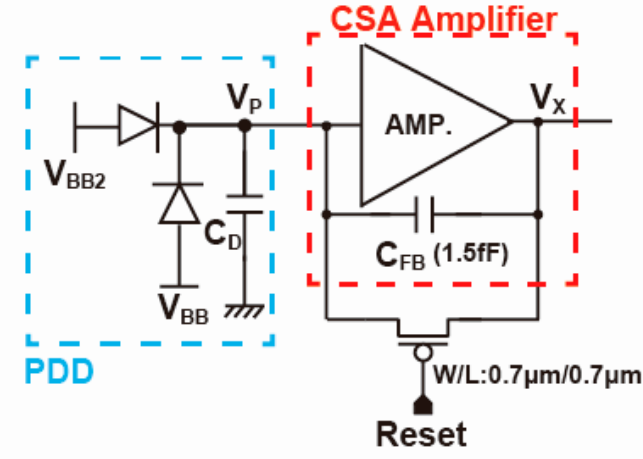

(a)

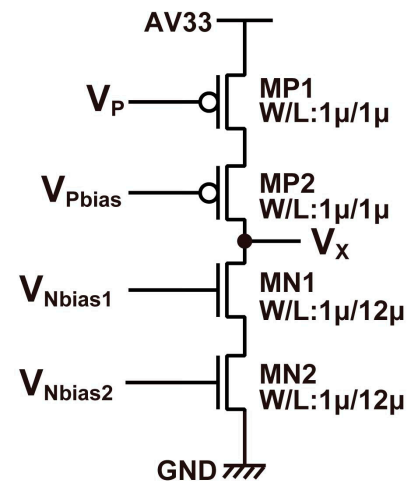

(b)

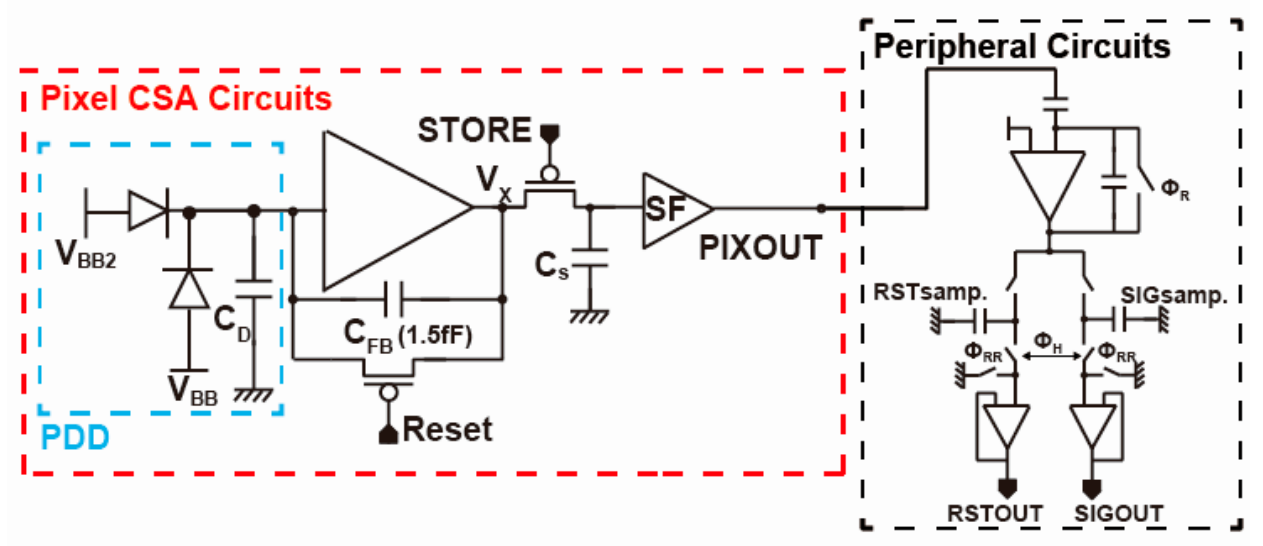

(c)

Figure 9. Equivalent circuits of the CSA in the pixel. (a) Model of CSA including those for the substrate detector, (b) internal amplifier, (c) Equivalent readout circuits chain including the pixel and peripheral.

The conversion gain is solely determined by $C_{F B}$ with a large gain internal amplifier, and therefore a very sensitive CSA is realized if $C_{F B}$ is designed to be very small.

The timing for the pixel operation is shown in Figure 10. The CSA with the pinned depleted diode detector (PDD) can be used for an event-driven type pixel using an in-pixel comparator as used in [9]. To evaluate the detector's basic characteristics, a simple integration type of operation is used here. A PMOS reset transistor is used for better dynamic range. After the reset switch is turned off, there is a 
charge injection from the reset transistor. The charge injection by the reset transistor is controlled by a proper choice of the transistor size and low-level voltage applied to the reset transistor. The operating point of the amplifier is shifted to relatively low level of $0.9 \mathrm{~V}$ by the charge injection at the output of the amplifier.

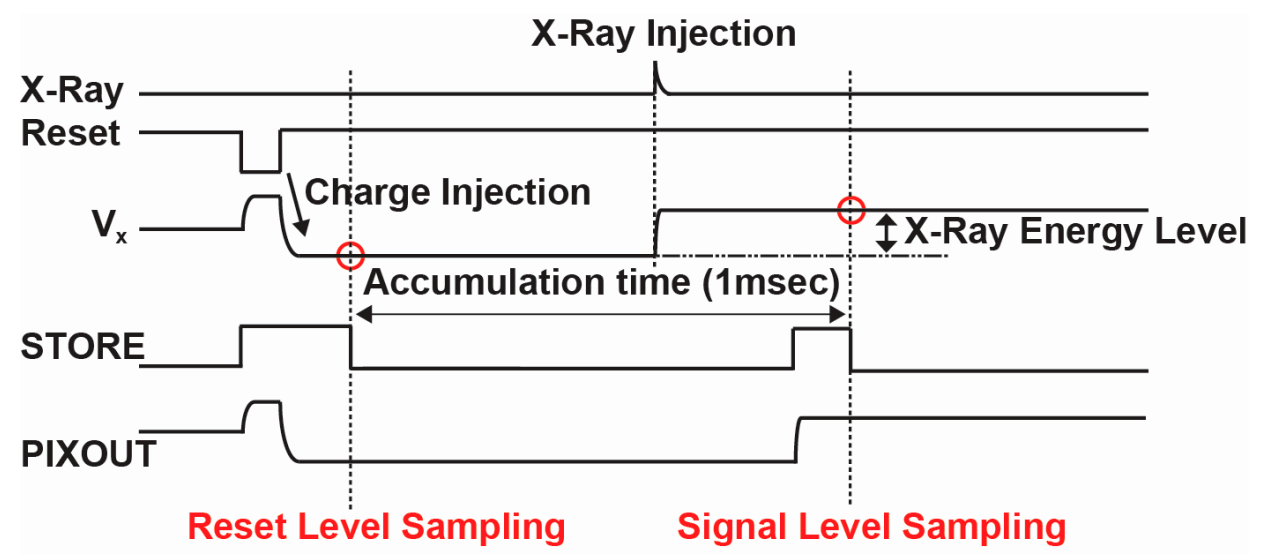

Figure 10. Pixel Timing Diagram.

After the reset operation, the reset level of the CSA output is sampled at a sample-and-hold capacitor $C_{S}$, and then the detector (and the CSA) waits an event of X-ray injection during the accumulation time shown in Figure 10. After that, the signal level of the CSA output is sampled at $C_{S}$ again. Using a switched-capacitor CDS circuit in the peripheral circuits, the CDS (correlated double sampling) for cancelling the reset noise of the CSA can be carried out. To do this, the reset and signal levels of the CSA output sampled in the $\mathrm{C}_{\mathrm{S}}$ are read out to the peripheral CDS circuit.

The use of very small capacitance of $C_{F B}$ and the resulting high conversion gain are effective for reducing the noises superimposed after the CSA such as those of an in-pixel source follower buffer, peripheral readout circuits, output buffer amplifier, and A-to-D converter. Then, the noise of the CSA is dominated by the capacitance of the charge sensing node of the PDD detector and the design of the internal amplifier. The input-referred noise of the designed CSA shown in Figure 9 is approximately expressed as:

$$
N_{n}=\frac{\sqrt{2}}{G_{C}} \sqrt{\frac{1}{\beta_{F}} \frac{\xi_{A} k_{B} T}{C_{S}}+\frac{N_{f}}{\beta_{F}^{2}}\left(\varepsilon+\ln \frac{T_{C D S}}{\tau_{C S A}}\right)}
$$

where $\xi_{A}$ is the excess thermal noise factor of the internal amplifier, $T_{C D S}$ the time difference of the two samples in the correlated double sampling operation used in the reset noise cancelling of the CSA, $N_{f}$ the flicker noise coefficient of the input transistor of the internal amplifier, $\varepsilon=0.577 \ldots$ Euler's constant, $k_{B}$ the Boltzmann's constant and T the absolute temperature, $\beta_{F}$ the feedback factor of the CSA given by:

$$
\beta_{F}=\frac{C_{F B}}{C_{F B}+C_{D}+C_{I}}
$$

and $\tau_{C S A}$ the time constant that determines the response time of the CSA which is given by

$$
\tau_{C S A}=\frac{C_{S}}{g_{m A} \beta_{F}}
$$

where $g_{m A}$ is the trans-conductance of the internal amplifier $[18,19]$. The first and second terms in the square root of Equation (4) is due to the thermal and flicker (1/f) noises of the CSA, respectively. Equation (4) indicates that the noise is much dependent on $\beta_{F}$ or the ratio of $C_{F B}$ to $C_{D}+C_{I}$. For a low-noise CSA, the reduction of $C_{D}$ and $C_{I}$ is very important while using small $C_{F B}$ for high conversion gain. The effort for highly-sensitive substrate detector described in Section 2 reduces $C_{D}$. The input 
capacitance of the internal amplifier $C_{I}$ is inversely proportional to the size (channel length (L) times channel width $(\mathrm{W})$ ) of input transistor (MP1 of Figure 9b). The flicker noise coefficient $N_{f}$ is also inversely proportional to the size (LW) of MP1 if the noises due to other transistors (MP2, MN2, and MN3) are not influenced. Therefore there exists an optimal choice of the size of MP1 to minimize the input-referred noise depending on $C_{D}, C_{F B}$ and other parameters that influence Equation (4). Figure 11 shows calculated input-referred noise as a function of the transistor size of MP1 with $C_{D}$ as a parameter. In this calculation, $C_{F B}=1.5[\mathrm{fF}], \mathrm{T}_{\mathrm{CDS}}=1[\mathrm{~ms}], \tau_{C S A}=0.2 \mu \mathrm{s}, \mathrm{C}_{S}=240[\mathrm{fF}]$ and other parameters are picked up by the PDK of the $0.2 \mu \mathrm{m}$ SOI technology. According to the simulation results of the PDD detector, $C_{D}$ is estimated to be $3.2 \mathrm{fF}$. From Figure 11 and with the optimum transistor size $\left(\mathrm{WL}=0.36 \mu \mathrm{m}^{2}\right)$, the noise level of $4.1 \mathrm{e}^{-} \mathrm{rms}$. is expected. However, this noise is critically increased if the flicker noise of transistors is larger than that used in this calculation. The use of larger transistor size leads to lower flicker noise and robust to noise increase. The design of the CSA for implementation uses $\mathrm{WL}=1.0 \mu \mathrm{m}^{2}$ for MP1 and the expected noise level is $4.5 \mathrm{e}^{-}$rms.

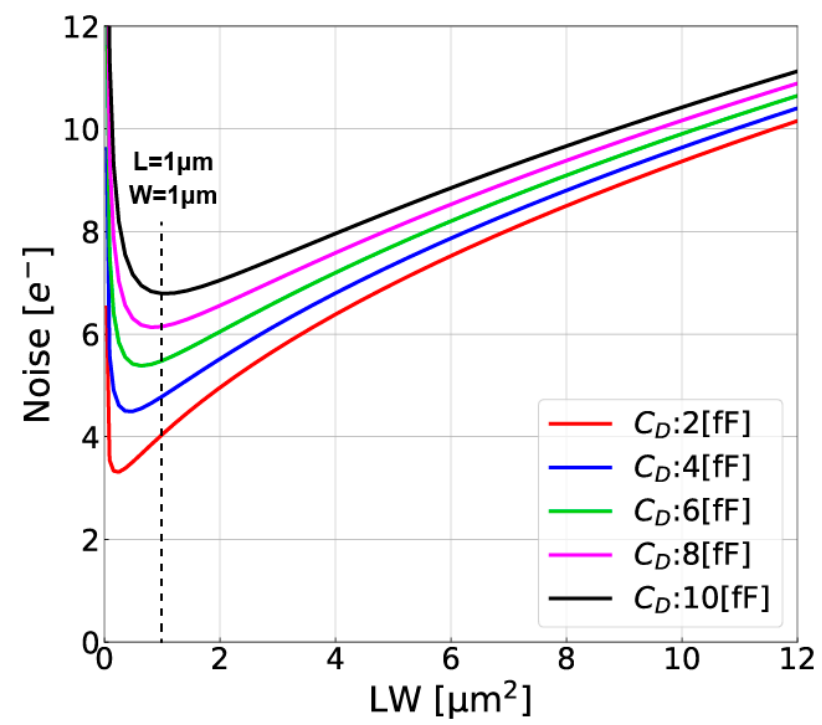

Figure 11. Input-referred Noise as a Function of LW of MP1 and $C_{D}$.

\section{Implementation and Measurements}

An experimental chip to evaluate the pixel performance of the SOIPIX-PDD was manufactured using $0.2 \mu \mathrm{m}$ SOI technology as summarized in Table 1. Figure 12a shows the chip microphotograph of the sensor chip. The chip includes $6 \times 6=36$ types of pixel arrays each of which has $8(\mathrm{~V}) \times 7(\mathrm{H})$ pixels and respective readout circuits as shown in Figure $12 \mathrm{~b}$. The pixel size is $36 \mu \mathrm{m} \times 36 \mu \mathrm{m}$. All the circuits and test elements are implemented in the chip die size of $4.45 \mathrm{~mm} \times 4.45 \mathrm{~mm}$. In the following measurement results, the $8 \times 7$ pixel array of a standard design whose detector dimensions and circuit parameters are described in Section 3 is used.

Table 1. SOIPIX Process Technology.

\begin{tabular}{|c|c|}
\hline Process & $\begin{array}{c}0.20 \mu \mathrm{m} \text { FD-SOI CMOS Technology with } \\
\text { Substrate-Detector Process }\end{array}$ \\
\hline Substrate thickness & $200 \mu \mathrm{m}$ \\
\hline Wafer type & FZ-p (Floating Zone, p-type) \\
\hline Substrate Resistivity & $>25 \mathrm{k} \Omega \mathrm{cm}$ \\
\hline
\end{tabular}




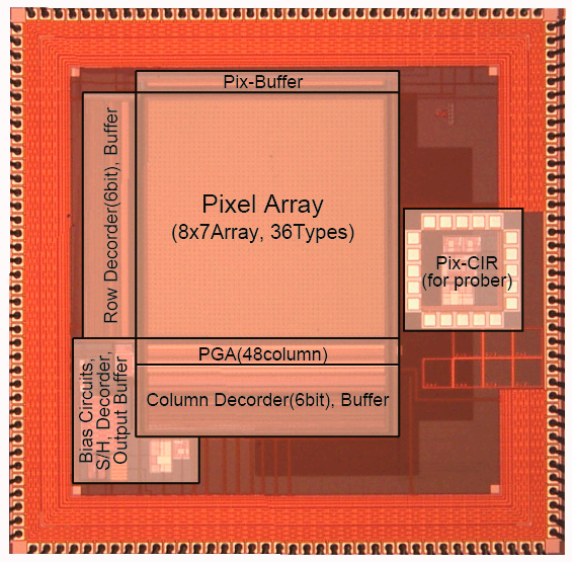

(a) Photo micrograph

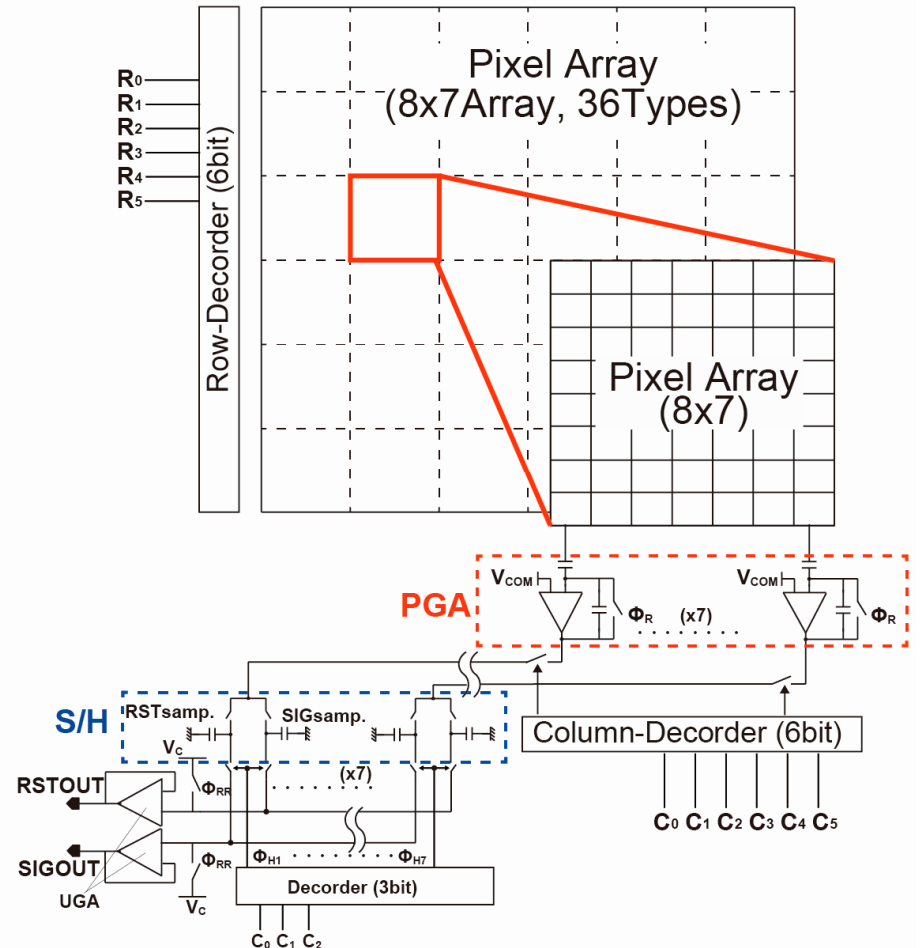

(b) Block diagram of the circuits

Figure 12. Implemented chip.

\subsection{Basic Characteristics of the SOI Pxel with Pnned Depleted Diode Structure}

Basic characteristics of the proposed pixel using the SOI technology with PDD structure as the substrate detector are measured. In the following measurement results if not stated, the applied backside bias $\left(\mathrm{V}_{\mathrm{BB}}\right)$ is $-60 \mathrm{~V}$, surface-side back-gate bias $\left(\mathrm{V}_{\mathrm{BB} 2}\right)$ is $-2 \mathrm{~V}$, the power supply voltage of the analog/digital pixel circuits is $3 \mathrm{~V}$. The pixel location of $(x=2, y=5)$ if not stated is picked up for the pixel characterization because the average noise level is obtained at this pixel as shown in Figure 17 below.

The implemented pixel has event-driven circuits using a comparator and logic gates together with the charge amplifier and analog readout circuits for the case of testing an event-driven type of X-ray energy spectrum measurements [20]. In the measurement results throughout this paper, however, the function of event detection and event-driven measurements is not used because the scope of this paper is to characterize the basic pixel performance.

\subsubsection{Linearity and Conversion Gain}

Figure 13 shows the linearity measurement of the pixel for the two backside biases $\left(\mathrm{V}_{\mathrm{BB}}\right)$ of $-10 \mathrm{~V}$ and $-60 \mathrm{~V}$. A white light generated and intensity-scanned by an LB-8611A precision lighting box (Kyoritsu, Tokyo, Japan) is used for the linearity measurements. The light is illuminated from the backside of the chip. With the thick $(200 \mu \mathrm{m})$ high-resistivity $(25 \mathrm{k} \Omega \mathrm{cm})$ substrate, the substrate is fully depleted by $\left|V_{\mathrm{BB}}\right|$ of higher than $14.4 \mathrm{~V}$. For $\mathrm{V}_{\mathrm{BB}}$ of $-10 \mathrm{~V}$, the linearity and sensitivity is poor because of incomplete depletion of the substrate. With a fully-depleted biasing of $\mathrm{V}_{\mathrm{BB}}=-60 \mathrm{~V}$, a good linearity is obtained in the output range up to $0.6 \mathrm{~V}$.

Figure 14 shows the noise as a function of signal amplitude for the measurement of conversion gain. Photon shot noise is used for the measurement of the conversion gain [21]. From the cross point of signal voltage and shot noise voltage, the conversion gain is measured to be $70 \mu \mathrm{V} / \mathrm{e}^{-}$. 


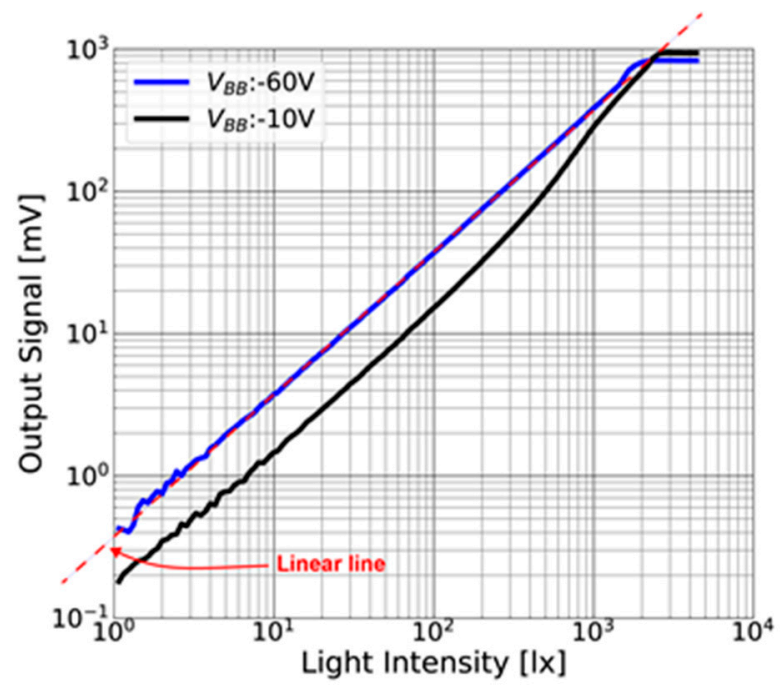

Figure 13. Linearity of the pixel output to light intensity.

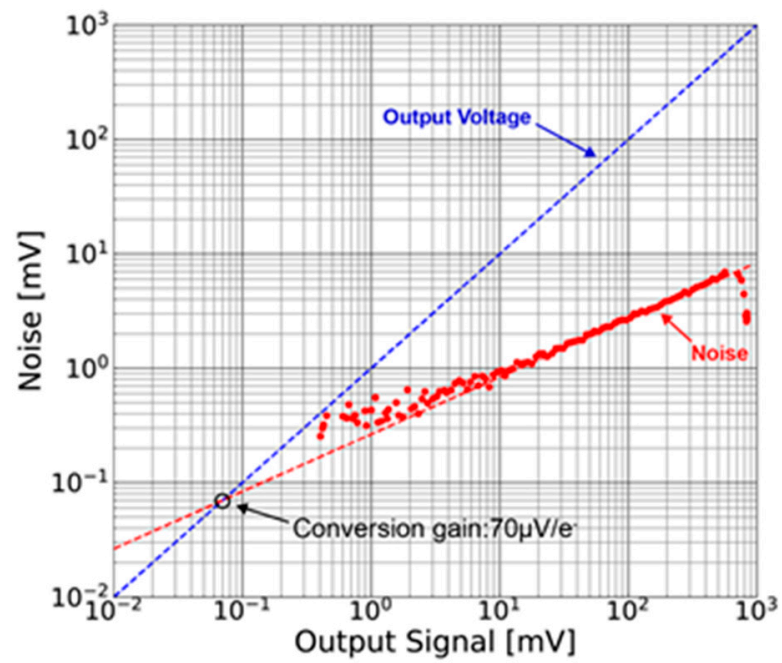

Figure 14. Noise versus signal for conversion gain measurement.

\subsubsection{Dark Current}

Figure 15 shows the temperature dependence of dark current of the SOIPIX-PDD. The dark current of the conventional SOI pixel [9] is also shown for comparison. For the comparison at ambient temperature of around $25^{\circ} \mathrm{C}$, the SOIPIX-PDD has 100 times smaller dark current density than that of the conventional SOI pixel. This shows the effectiveness of the pinned depleted diode structure using neutralized BPW layer created just under the BOX to fill the surface with holes for the reduction of the dark current. For the temperature range of higher than $5{ }^{\circ} \mathrm{C}$, or smaller than $3.6 / 1000[1 / \mathrm{K}]$ in the Arrhenius plot, it follows the line that the activation energy of dark current is $0.56 \mathrm{eV}$, the half of band gap $(=1.12 \mathrm{eV})$ of silicon, indicating that the dominant dark current component is still due to SRH generation current of the detector. For the temperature range of smaller than $5^{\circ} \mathrm{C}$, or larger than 3.6/1000 $[1 / \mathrm{K}]$ in the Arrhenius plot, the temperature dependency tends to saturate and it takes $56\left[\mathrm{pA} / \mathrm{cm}^{2}\right]$ at $-35^{\circ} \mathrm{C}$. The reason for this limitation of the dark current reduction at low temperature is not clear at the moment, but the possible reason is a leakage current of the p-MOS transistor used for resetting the sensing node. This is because the trap-assisted band-to-band tunneling, which is often a major mechanism of leakage current of MOS transistor, has small temperature dependence. 


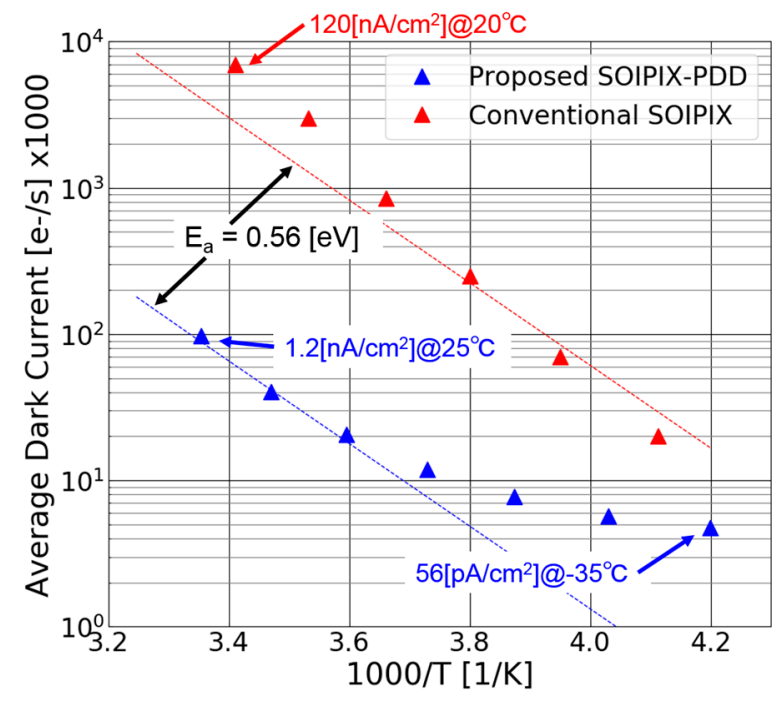

Figure 15. Temperature dependence of dark current.

Figure 16 shows a map of distribution of the dark current measured at $25^{\circ} \mathrm{C}$ and $-35^{\circ} \mathrm{C}$. The mean and standard deviation are $1200\left[\mathrm{pA} / \mathrm{cm}^{2}\right]$ and $51.6\left[\mathrm{pA} / \mathrm{cm}^{2}\right](=4.3 \%$ of mean value $)$ at $25^{\circ} \mathrm{C}$ and and $56\left[\mathrm{pA} / \mathrm{cm}^{2}\right]$ and $6.3\left[\mathrm{pA} / \mathrm{cm}^{2}\right](=12.6 \%$ of mean value $)$ at $-35^{\circ} \mathrm{C}$.

\begin{tabular}{|c|c|c|c|c|c|c|c|}
\hline$y$ & 1 & 2 & 3 & 4 & 5 & 6 & 7 \\
\hline 1 & 1310 & 1160 & 1190 & 1230 & 1160 & 1160 & 1100 \\
\hline 2 & 1270 & 1210 & 1250 & 1200 & 1160 & 1200 & 1130 \\
\hline 3 & 1270 & 1170 & 1210 & 1180 & 1170 & 1150 & 1210 \\
\hline 4 & 1290 & 1220 & 1210 & 1220 & 1190 & 1180 & 1160 \\
\hline 5 & 1310 & 1200 & 1230 & 1230 & 1180 & 1160 & 1150 \\
\hline 6 & 1290 & 1240 & 1240 & 1200 & 1250 & 1180 & 1100 \\
\hline 7 & 1300 & 1230 & 1220 & 1260 & 1190 & 1180 & 1120 \\
\hline 8 & 1300 & 1220 & 1230 & 1230 & 1170 & 1200 & 1100 \\
\hline
\end{tabular}

(a) at $25^{\circ} \mathrm{C}$

\begin{tabular}{|c|c|c|c|c|c|c|c|}
\hline$x$ & 1 & 2 & 3 & 4 & 5 & 6 & 7 \\
\hline 1 & 50 & 38 & 50 & 56 & 46 & 48 & 40 \\
\hline 2 & 54 & 45 & 48 & 49 & 50 & 48 & 40 \\
\hline 3 & 49 & 50 & 50 & 50 & 50 & 41 & 41 \\
\hline 4 & 58 & 59 & 65 & 49 & 50 & 41 & 52 \\
\hline 5 & 56 & 56 & 49 & 48 & 56 & 44 & 40 \\
\hline 6 & 55 & 52 & 50 & 42 & 51 & 46 & 40 \\
\hline 7 & 66 & 53 & 48 & 57 & 54 & 47 & 36 \\
\hline 8 & 58 & 56 & 50 & 56 & 51 & 55 & 47 \\
\hline
\end{tabular}

(b) at $-35^{\circ} \mathrm{C}$

Figure 16. Pixel-to-pixel deviation of dark current.

\subsubsection{Readout Noise}

Figure 17 shows measured input-referred readout noise of all the $8 \times 7$ pixels. The average value of the noise is $11.0 \mathrm{e}^{-}$rms and the minimum and maximum noises are $8.6 \mathrm{e}^{-}$rms and $14.3 \mathrm{e}^{-}$rms, respectively. Compared with the conventional SOI pixel whose readout noise is $35 \mathrm{e}^{-} \mathrm{rms}$ [9], the readout noise of the SOIPIX-PDD is reduced to one-third of that. The readout noise calculated by Equation (4) and designed parameters $\left.\left(C_{F B}=1.5[\mathrm{fF}], T_{C D S}=1[\mathrm{~ms}], \tau_{C S A}=0.2 \mu \mathrm{s}, \mathrm{CS}=240[\mathrm{fF}]\right)\right)$ is $4.5 \mathrm{e}^{-} \mathrm{rms}$ at $107 \mu \mathrm{V} / \mathrm{e}^{-}$. With the measured conversion gain in Figure 14 , i.e., $70 \mu \mathrm{V} / \mathrm{e}^{-}$, the readout noise using Equation (4) is calculated to be $5.8 \mathrm{e}^{-} \mathrm{rms}$ if $\mathrm{CFB}=2.3$ [fF]. In any way, measured readout noise is bigger than calculated noise. The reason for increased readout noise is considered to be an increase in $C_{D}$, 
an increase in $\mathrm{C}_{\mathrm{FB}}$ due to parasitic capacitance, and coupling of power supply noise through power and ground lines and substrates.

\begin{tabular}{|c|c|c|c|c|c|c|c|}
\hline$y$ & 1 & 2 & 3 & 4 & 5 & 6 & 7 \\
\hline 1 & 9.6 & 8.6 & 9.3 & 10.3 & 10.7 & 10.0 & 13.9 \\
\hline 2 & 10.6 & 9.0 & 9.7 & 10.8 & 10.8 & 10.4 & 13.2 \\
\hline 3 & 9.2 & 10.9 & 11.1 & 11.0 & 10.6 & 10.2 & 12.6 \\
\hline 4 & 11.7 & 13.3 & 13.2 & 9.8 & 10.7 & 11.4 & 13.7 \\
\hline 5 & 11.1 & 11.0 & 11.7 & 10.1 & 10.9 & 9.7 & 13.4 \\
\hline 6 & 10.3 & 11.2 & 10.0 & 9.1 & 10.5 & 10.1 & 12.7 \\
\hline 7 & 12.3 & 11.3 & 9.8 & 10.0 & 11.1 & 11.3 & 14.3 \\
\hline 8 & 11.6 & 11.1 & 10.4 & 10.7 & 10.7 & 10.6 & 12.6 \\
\hline
\end{tabular}

Figure 17. Map of input referred noise $\left[\mathrm{e}^{-}\right.$rms $]$of an $8 \times 7$ pixel array with cooling at $-35{ }^{\circ} \mathrm{C}$. Highlighted $3 \times 3$ pixel array is used for X-ray energy spectrum measurement. A pixel $(x=2, y=5)$ shown by red-colored box is used for the measurement of single pixel events. Adjacent 8 pixels shown by yellow-colored boxes are used for eliminating events of charge splitting to plural of pixels.

\subsection{X-ray Eergy Sectrum}

To evaluate the pixel performance for X-ray energy spectrum measurement, a particular pixel $(x=2, y=5)$ of the $8 \times 7$ pixel array is used for the measurement of single pixel events. The adjacent eight pixels are used for eliminating events of charge splitting to plural of pixels. To do this, if the pixel for spectrum measurement has an event by checking whether if the signal is larger than the event threshold, and only if the signals of all adjacent eight pixels are smaller than a given threshold, i.e., the split threshold, the event is counted as the measured energy level in the spectrum. Figure 18 shows the ${ }^{241} \mathrm{Am} X$-ray spectra of single pixel events obtained with the SOIPIX-PDD chip after the data reduction and analyses given in [20]. Since the measurement system uses a 14-bit A/D converter with the analog range of $2 \mathrm{~V}, 1$ ADU corresponds to a bin of $122 \mu \mathrm{V}$ and an energy bin of $6.54 \mathrm{eV}$ with the conversion gain of $70 \mu \mathrm{V} / \mathrm{e}^{-}$and $\omega$ (= energy required to liberate one electron-hole pair) of $3.65 \mathrm{eV} / \mathrm{e}^{-}$. Figure 18. In this measurement, the X-ray event only at the pixel $(x=2, y=5)$ and adjacent 8 pixels shown by yellow-colored zone in Figure 17 is considered for the evaluation of single pixel events. The energy resolution of the SOIPIX-PDD is $280 \mathrm{eV} \mathrm{(2.01 \% )} \mathrm{in} \mathrm{FWHM} \mathrm{at} 13.95 \mathrm{keV}$. In this measurement, the event threshold, split threshold and energy bin of the spectrum are set to $10 \mathrm{ADU}(=65 \mathrm{eV}), 10 \mathrm{ADU}(=65 \mathrm{eV})$ and $1.3 \mathrm{ADU}(=8.5 \mathrm{eV})$, respectively. In the conventional SOIPIX, as given in [20], the FWHM of $1500 \mathrm{eV}(10.8 \%)$ at $13.95 \mathrm{keV}$ of the ${ }^{241} \mathrm{Am}$ was obtained, indicating the effectiveness of the SOIPIX-PDD for improving the energy resolution by a factor of more than 5 . Another good effect in the SOIPIX-PDD when compared with the conventional SOI pixels is the very-small tailing structures of the energy spectrum to lower-energy side which is possibly caused by the signal charge loss in the sensor layers [9]. The result of Figure 18 shows the proposed detector has high charge collection efficiency or small signal charge loss thanks to the employment of the pinned depleted diode structure. The theoretical limit of energy resolution (FWHM) of a detector can be found using:

$$
\Delta E(e V)=2.354 \omega \sqrt{\frac{F E}{\omega}+\sigma^{2}}
$$


where $F$ is the Fano factor ( 0.11 for silicon), $E$ is the energy of the incident $X$-ray photon, and $\sigma$ is measured readout noise. With the measured noise of the pixel $(\mathrm{x}=2, \mathrm{y}=5)$, i.e., $11.0 \mathrm{e}^{-}$, the energy resolution using Equation (7) is calculated to be $200 \mathrm{eV}(1.43 \%)$ at $\mathrm{E}=13.95 \mathrm{keV}$. Therefore, there is a factor for further improvement of the energy resolution other than the readout noise. In the measurement of Figure 18, because the radiation source irradiates from the surface side of the SOI pixel, there still exists a possibility of signal charge loss due to the recombination in the neutral BPW layer of the SOIPIX-PDD detector.

Figure 19 demonstrates the conversion gain of $70 \mu \mathrm{V} / \mathrm{e}^{-}$obtained by shot noise measurement to agree with the measured signal voltages to the ${ }^{241} \mathrm{Am}$ 's characteristic X-ray lines of $13.95,17.74$, 20.8 and $26.3 \mathrm{keV}$. In Figure 18, a spectral peak probably due to the characteristic X-ray line of $59.5 \mathrm{keV}$ is observed at the pulse height of $7700 \mathrm{ADU}$. This pulse height corresponds to $0.94 \mathrm{~V}$ as the output signal voltage, while the implemented pixel has the output voltage linearity up to $0.6 \mathrm{~V}$ as shown in Figure 13. The characteristic X-ray line of $59.5 \mathrm{keV}$ cannot be exactly measured because the pulse height is outside of the linear range of the designed detector. Therefore the data point for $59.5 \mathrm{keV}$ is not included in Figure 19.

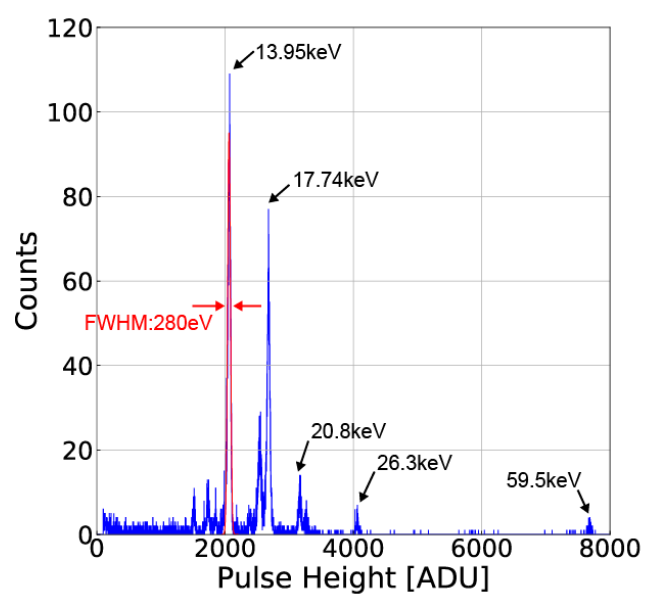

Figure 18. Measured X-ray spectra of ${ }^{241} \mathrm{Am}$ as single-pixel events using the SOIPIX-PDD. $1 \mathrm{ADU}$ is $122 \mu \mathrm{V} / \mathrm{e}^{-}$(2 V/14 bit).

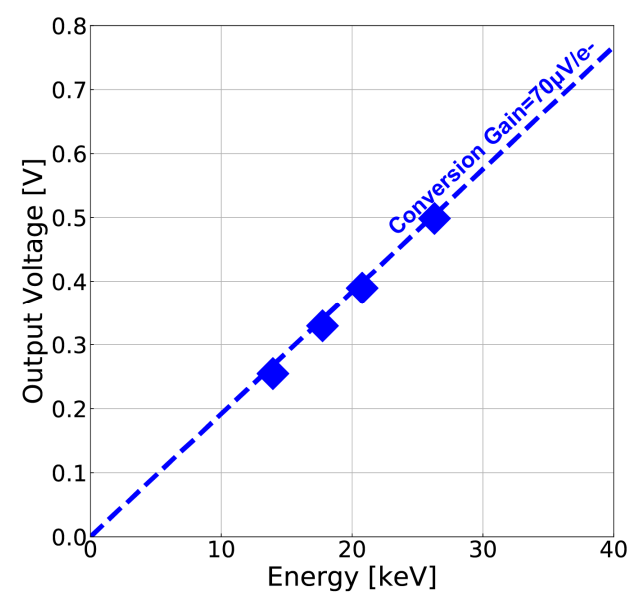

Figure 19. Confirmation of conversion gain with the measured X-ray spectra of ${ }^{241} \mathrm{Am}$.

Figure 20 shows the ${ }^{55} \mathrm{Fe} X$-ray spectra of single pixel events using the $\operatorname{pixel}(\mathrm{x}=2, \mathrm{y}=2)$ of the SOIPIX-PDD detector of a different chip from that used for measurement of Figures 13-19. The bias voltage of $\mathrm{V}_{\mathrm{BB}}$ and $\mathrm{V}_{\mathrm{BB} 2}$ are set at $-60 \mathrm{~V}$, and $-2.7 \mathrm{~V}$, respectively. A very good energy resolution 
(FWHM) of $200 \mathrm{eV}(3.6 \%)$ at $5.9 \mathrm{keV}$ and very small tailing are obtained. The Mn-K (5.9 keV) and Mn-K $(6.4 \mathrm{keV})$ lines are definitely discriminated.

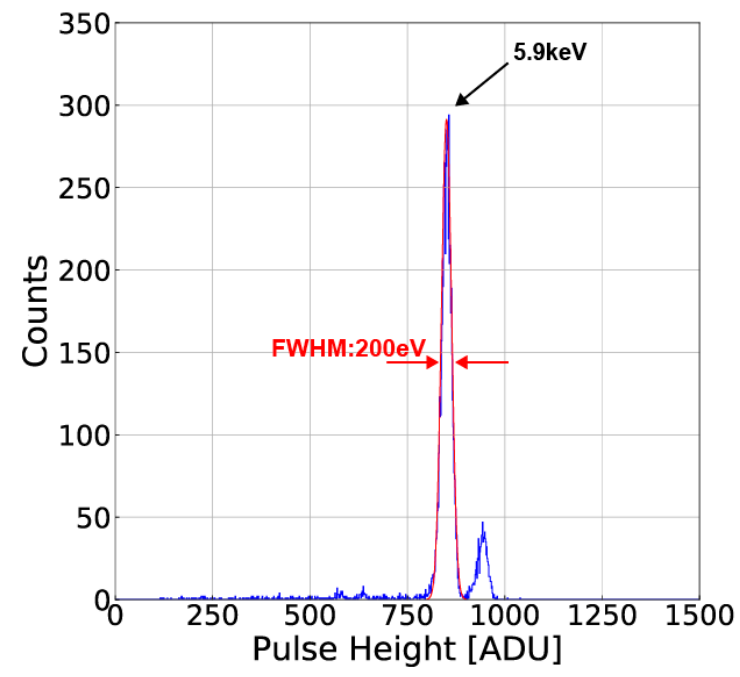

Figure 20. Measured X-ray spectra of ${ }^{55} \mathrm{Fe}$ as single-pixel events using the SOIPIX-PDD.

\subsection{Performance Comparison}

Table 2 shows a comparison of the conventional SOIPIX (XRPIX series) and SOIPIX-PDD. Using SOIPIX-PDD, the readout noise and dark current are significantly reduced and the resulting X-ray spectroscopic performance greatly improved when compared with the conventional SOIPIX detectors. Though it is not shown in the Table 2, characteristic X-ray spectral line with very small tailing because of high charge collection efficiency is another advantage of the SOIPIX-PDD.

Table 2. Comparison with the conventional SOIPIX (XRPIX series) and SOIPIX-PDD.

\begin{tabular}{ccccc}
\hline SOIPIX Type & XRPIX1 [20] & XRPIX2b-A [9] & XRPIX3b-CSA [9] & SOIPIX-PDD \\
\hline Conversion gain & 3.56 & 7.0 & 17.8 & 70 \\
\hline Readout noise & $129 \mathrm{e}^{-} \mathrm{rms}$ & $68 \mathrm{e}^{-} \mathrm{rms}$ & $35 \mathrm{e}^{-} \mathrm{rms}$ & $11.0 \mathrm{e}^{-} \mathrm{rms}^{-}$ \\
\hline Dark current & N. A. & N. A. & $120 \mathrm{nA} / \mathrm{cm}^{2} @ 25^{\circ} \mathrm{C}$ & $1.2 \mathrm{nA} / \mathrm{cm}^{2} @ 20^{\circ} \mathrm{C}$ \\
\hline $\begin{array}{c}\text { Energy resolution } \\
\text { (FWHM@5.9 keV) }\end{array}$ & N. A. & N. A. & $320 \mathrm{eV} \mathrm{(5.4 \% )}$ & $200 \mathrm{eV} \mathrm{(3.6 \% )}$ \\
\hline $\begin{array}{c}\text { Energy resolution } \\
\text { (FWHM@13.95 keV) }\end{array}$ & $1500 \mathrm{eV}(10.8 \%)$ & $(\sim 1500 \mathrm{eV})^{*}$ & N. A. & $280 \mathrm{eV}(2.0 \%)$ \\
\hline
\end{tabular}

* Numerical value of energy resolution is not reported, but it is estimated from the graph of X-ray energy spectra for ${ }^{241}$ Am given in [9].

\section{Conclusions}

A novel SOI pixel detector using a pinned depleted diode structure (SOIPIX-PDD) has been presented in this paper. The SOIPIX-PDD realizes a low readout noise due to small charge sensing node capacitance, low dark current due to a pinned $\mathrm{Si}$ surface at $\mathrm{Si}-\mathrm{SiO}_{2}$ interface of the detector under the BOX layer and high charge collection efficiency with the buried channel for carrier collection. The implemented chip demonstrates that the SOIPIX-PDD pixels has a high-conversion gain of $70 \mu \mathrm{V} / \mathrm{e}^{-}$, low noise of $11.0 \mathrm{e}^{-}$rms, low dark current of $56 \mathrm{pA} / \mathrm{cm}^{2}$ at $-35^{\circ} \mathrm{C}$, and good energy resolution in the measured characteristic X-ray lines, e.g., 200eV(FWHM) at $5.9 \mathrm{keV}$ and $280 \mathrm{eV}$ (FWHM) at $13.95 \mathrm{keV}$. 
Acknowledgments: We acknowledge the valuable advice and great work by the personnel of LAPIS Semiconductor Co., Ltd. This study was supported by the Japan Society for the Promotion of Science (JSPS) KAKENHI Grant-in-Aid for Scientific Research on Innovative Areas 25109002 (Y.A.), 25109003 (S.K.), 25109004 (T.G.T.) and Grand-in-Aid for Young Scientists (B) 15K17648 (A.T.). This study was also supported by the VLSI Design and Education Center (VDEC), the University of Tokyo in collaboration with Cadence Design Systems, Inc., and Mentor Graphics, Inc. The authors would like to thank Nobukazu Teranishi and Keiichiro Kagawa of Shizuoka University and Ikuo Kurachi of High Energy Accelerator Research Organization (KEK) for helpful discussion.

Author Contributions: Shoji Kawahito, Takeshi Go Tsuru and Yasuo Arai proposed the device concept and provided the overall guidance of the project. Hiroki Kamehama, Shoji Kawahito, Sumeet Shrestha, Keita Yasutomi, Ayakai Takeda and Takeshi Go Tsuru designed the detector. Hiroki Kamehama and Syunta Nakanishi and Takeshi Go Tsuru measured the chip. Hiroki Kamehama and Shoji Kawahito drafted the manuscript.

Conflicts of Interest: The authors declare no conflict of interest.

\section{References}

1. Koyama, K.; Tsunemi, H.; Dotani, T.; Bautz, M.W.; Hyashida, K.; Tsuru, T.G.; Matsumoto, H.; Ogawara, Y.; Ricker, G.R.; Doty, J.; et al. X-ray imaging spectrometer (XIS) on board Suzaku. Publ. Astron. Soc. Jpn. 2015, 59, 23-33. [CrossRef]

2. Garmire, G.P.; Bautz, M.W.; Ford, P.G.; Nousek, J.A.; Ricker, G.R., Jr. Advanced CCD Imaging Spectrometer (ASICS) instrument on the Chandra X-ray Observatory. In X-ray and Gamma-ray Telescopes and Instruments for Astronomy; Trumper, J.E., Tananbaum, H.D., Eds.; SPIE: Bellingham, WA, USA, 2003; pp. $28-44$.

3. Arai, Y.; Miyoshi, T.; Unno, Y.; Tsuboyama, T.; Terada, S.; Ikegami, Y.; Ichimiya, R.; Kohriki, T.; Tauchi, K.; Ikemoto, Y.; et al. Development of SOI pixel process technology. In Nuclear Instruments and Methods in Physics Research Section A: Accelerators, Spectrometers, Detectors and Associated Equipment; Elsevier: Amsterdam, The Netherlands, 2011; Volume 636, pp. S31-S36.

4. Griffith, C.V.; Falcon, A.D.; Prieskorn, Z.R.; Burrows, D.N. Speedster-EXD: A new event-driven hybrid CMOS X-ray detector. J. Astron. Telesc. Inst. 2016, 2, 1-13. [CrossRef]

5. Arai, Y.; Miyoshi, T.; Unno, Y.; Tsuboyama, T.; Terada, S.; Ikegami, Y.; Kohriki, T.; Tauchi, K.; Ilemoto, Y.; Ichimiya, R.; Ikeda, H.; et al. Development of SOI monolithic pixel detectors. In Nuclear Instruments and Methods in Physics Research Section A: Accelerators, Spectrometers, Detectors and Associated Equipment; Elsevier: Amsterdam, The Netherlands, 2010; Volume 623, pp. 186-188.

6. Nakashima, S.; Ryu, S.G.; Tsuru, T.G.; Takeda, A.; Arai, Y.; Miyoshi, T.; Ichimiya, R.; Ikemoto, Y.; Imamura, T.; Ohmoto, T.; et al. Progress in development of monolithic active pixel detector for X-ray astronomy with SOI CMOS technology. Phys. Procedia 2012, 37, 1373-1380. [CrossRef]

7. Tsuru, T.G.; Matsumura, H.; Takeda, A.; Tanaka, T.; Nakashima, S.; Arai, Y.; Mori, K.; Takenaka, R.; Nishioka, Y.; Takayoshi, K.; et al. Development and performance of Kyoto's X-ray astronomical SOI pixel (SOIPIX) sensor. In Space Telescopes and Instrumentation 2014: Ultraviolet to Gamma Ray; International Society for Optics and Photonics: Montreal, QC, Canada, 2014.

8. Takeda, A.; Arai, Y.; Ryu, S.G.; Nakashima, S.; Tsuru, T.G.; Imamura, T.; Ohmoto, T.; Iwata, A. Design and evaluation of an SOI pixel sensor for trigger-driven X-ray readout. IEEE Trans. Nucl. Sci. 2013, 60, 586-591. [CrossRef]

9. Takeda, A.; Tsuru, T.G.; Tanaka, T.; Uchida, H.; Matsumura, H.; Arai, Y.; Mori, K.; Nishioka, Y.; Takenaka, R.; Kohmura, T. Improvement of spectroscopic performance using a charge-sensitive amplifier circuit for an X-ray astronomical SOI pixel detector. J. Instrum. 2015, 10, C06005. [CrossRef]

10. Okihara, M.; Kasai, H.; Miura, N.; Kuriyama, N.; Nagatomo, Y.; Hatsui, T.; Omodani, M.; Miyoshi, T.; Arai, Y. Progress of FD-SOI technology for monolithic pixel detectors. In Proceedings of the 2012 IEEE Nuclear Science Symposium and Medical Imaging Conference (NSS/MIC), Anaheim, CA, USA, 27 October-3 November 2012; pp. 471-474.

11. Hashimoto, R.; Arai, Y.; Igarashi, N.; Kumai, R.; Miyoshi, T.; Kishimoto, S. Test results of a counting type SOI device for a new X-ray area detector. In Proceedings of the AIP Conference; American Institute of Physics: Melville, NY, USA, 2016; p. 1741.

12. Lauxtermann, S.; Vangapally, V. A Fully Depleted Backside Illuminated CMOS Imager with VGA Resolution and 15-micron Pixel Pitch. In Proceedings of the IEEE International Image Sensor Workshop, Snowbird, UT, USA, 12-16 June 2013. 
13. Hatsui, H.; Omodani, M.; Kudo, T.; Kobayashi, K.; Imamura, T.; Ohmoto, T.; Iwata, A.; Ono, S.; Kirihara, Y.; Kameshima, T.; et al. A direct detection X-ray CMOS image sensor with $500 \mu \mathrm{m}$ thick high resistivity silicon. In Proceedings of the IEEE International Image Sensor Workshop, Snowbird, UT, USA, 12-16 June 2013.

14. Sekiguchi, Y.; Arai, Y.; Hamagaki, H.; Gunji, T. Development of SOI pixel sensor for environmental radiation monitor. RIKEN Accel. Prog. Rep. 2014, 47, 231.

15. Fahim, F.; Deptuch, G.; Holm, S.; Shenai, A.; Lipton, R. Monolithic active pixel matrix with binary counters ASIC with nested wells. J. Instrum. 2013, 8, C04008. [CrossRef]

16. Kamehama, H.; Shrestha, S.; Yasutomi, K.; Kagawa, K.; Takeda, A.; Tsuru, T.G.; Arai, Y.; Kawahito, S. Fully depleted SOI pixel photo detectors with backgate surface potential pinning. In Proceedings of the IEEE International Image Sensor Workshop, Vaals, The Netherlands, 8-11 June 2015.

17. Teranishi, N.; Kohno, A.; Ishihara, Y.; Arai, K. No image lag photodiode structure in the interline CCD image sensor. In Proceedings of the International Electron Devices Meeting, San Francisco, CA, USA, 13-15 December 1982; pp. 324-327.

18. Kawahito, S.; Seo, M.W. Noise reduction effect of multiple-sampling-based signal-reduction circuits for ultra-low Noise CMOS image sensors. Sensors 2016, 16, 1867. [CrossRef] [PubMed]

19. Kawahito, S.; Itoh, S. Noise calculation model and analysis of high-gain readout circuits for CMOS image sensors. In Proceedings of the Electronic Imaging, San Jose, CA, USA, 28-31 January 2008.

20. Ryu, S.G.; Tsuru, T.G.; Nakashima, S.; Takeda, A.; Arai, Y.; Miyoshi, T.; Ichimiya, R.; Ikemoto, Y.; Matsumoto, H.; Imamura, T.; et al. First performance evaluation of an X-ray SOI pixel sensor for imaging spectroscopy and intra-pixel trigger. IEEE Trans. Nucl. Sci. 2011, 58, 2528-2536. [CrossRef]

21. Nakamura, J. (Ed.) Image Sensors and Signal Processing for Digital Still Camera; CRC: Boca Raton, FL, USA, 2005; pp. 81-83.

(C) 2017 by the authors. Licensee MDPI, Basel, Switzerland. This article is an open access article distributed under the terms and conditions of the Creative Commons Attribution (CC BY) license (http://creativecommons.org/licenses/by/4.0/). 